\title{
Investigating the Characteristics of Causal Relations in Japanese Text
}

\author{
Takashi Inui and Manabu Okumura \\ Precision and Intelligence Laboratory \\ Tokyo Institute of Technology \\ 4259, Nagatsuta, Midori-ku, Yokohama, 226-8503, Japan \\ tinui@lr.pi.titech.ac.jp,oku@pi.titech.ac.jp
}

\begin{abstract}
We investigated of the characteristics of in-text causal relations. We designed causal relation tags. With our designed tag set, three annotators annotated 750 Japanese newspaper articles. Then, using the annotated corpus, we investigated the causal relation instances from some viewpoints. Our quantitative study shows that what amount of causal relation instances are present, where these relation instances are present, and which types of linguistic expressions are used for expressing these relation instances in text.
\end{abstract}

\section{Introduction}

For many applications of natural language techniques such as question-answering systems and dialogue systems, acquiring knowledge about causal relations is one central issue. In recent researches, some automatic acquisition methods for causal knowledge have been proposed (Girju, 2003; Sato et al., 1999; Inui, 2004). They have used as knowledge resources a large amount of electric text documents: newspaper articles and Web documents.

To realize their knowledge acquisition methods accurately and efficiently, it is important to knowing the characteristics of presence of in-text causal relations. However, while the acquisition methods have been improved by some researches, the characteristics of presence of in-text causal relations are still unclear: we have no empirical study about what amount of causal relation instances exist in text and where in text causal relation instances tend to appear.

In this work, aiming to resolve the above issues, we create a corpus annotated with causal relation information which is useful for investigating what amount of causal relation instances are present and where these instances are present in text. Given some Japanese newspaper articles, we add our designed causal relation tags to the text segments. After creating the annotated corpus, we investigate the causal relation instances from three viewpoints: (i) cue phrase markers, (ii) part-of-speech information, and (iii) positions in sentences.

There are some pieces of previous work on analysis of in-text causal relations. However, although causal relation instances appear in several different ways, just a few forms have been treated in the previous studies: the verb phrase form with cue phrase markers such as in (1a) has been mainly treated. In contrast, we add our causal relation tags to several types of linguistic expressions with wide coverage to realize further analyses from above three points. Actually, we treat not only linguistic expressions with explicit cues such as in (1a), but also those without explicit cues, i.e. implicit, as in (1b), those formed by noun phrases as in (1c), and those formed between sentences as in (1d).

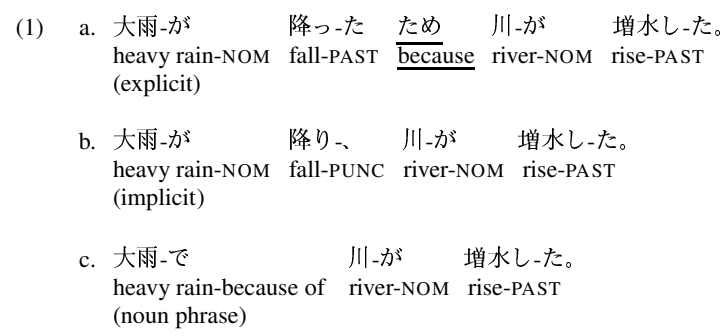




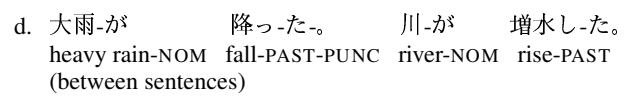

We apply new criteria for judging whether a linguistic expression includes a causal relation or not. Generally, it is hard to define rigorously the notion of causal relation. Therefore, in previous studies, there have been no standard common criteria for judging causal relations. Researchers have resorted to annotators' subjective judgements. Our criteria are represented in the form of linguistic templates which the annotators apply in making their judgements (see Section 3.2).

In Section 2, we will outline several previous research efforts on in-text causal relations. In Section 3 to Section 6, we will describe the details of the design of our causal relation tags and the annotation workflow. In Section 7, using the annotated corpus, we will then discuss the results for the investigation of characteristics of in-text causal relations.

\section{Related work}

Liu (2004) analyzed the differences of usages of some Japanese connectives marking causal relations. The results are useful for accounting for an appropriate connective for each context within the documents. However Liu conducted no quantitative studies.

Marcu (1997) investigated the frequency distribution of English connectives including "because" and "since" for implementation of rhetorical parsing. However, although Marcu's study was quantitative one, Marcu treated only explicit linguistic expressions with connectives. In the Timebank corpus (Pustejovsky et al., 2003), the causal relation information is included. However, the information is optional for implicit linguistic expressions.

Although both explicit expressions and implicit expressions are treated in the Penn Discourse Treebank (PDTB) corpus (Miltsakaki et al., 2004), no information on causal relations is contained in this corpus.

Altenberg (1984) investigated the frequency distribution of causal relation instances from some viewpoints such as document style and the syntactic form in English dialog data. Nishizawa (1997) also conducted a similar work using Japanese dialog data. Some parts of their viewpoints are overlapping with ours. However, while their studies focused on dialog data, our target is text documents. In fact, Altenberg treated also English text documents. However, our focus in this work is Japanese.

\section{Annotated information}

\subsection{Causal relation tags}

We use three tags head, mod, and causal_rel to represent the basic causal relation information. Our annotation scheme for events is similar to that of the PropBank (Palmer et al., 2005). An event is regarded as consisting of a head element and some modifiers. The tags head and mod are used to represent an event which forms one part of the two events held in a causal relation. The tag causal_rel is used to represent a causal relation between two annotated events.

Figure 1 shows an example of attaching the causal relation information to the sentence $(2 \mathrm{a})$, in which a causal relation is held between two events indicated (2b) and (2c) . Hereafter, we denote the former (cause) part of event as $e_{1}$ and the latter (effect) part of event as $e_{2}$.

（2） a. そして、遠方からの観光客がGWに入って増える。 (As the Golden Week holidays come, the number of sightseers from all over begins to increase.)

b. $e_{1}=\mathrm{GW}$ に入る

(The Golden Week holidays come)

c. $e_{2}=$ 遠方からの観光客が増える

(The number of sightseers from all over begins to increase)

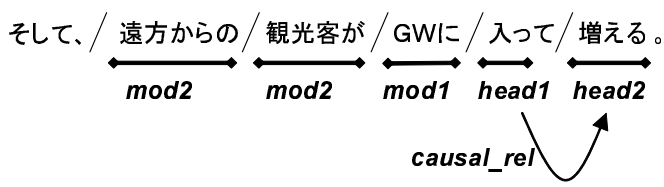

Figure 1: An example of attaching the causal relation information

The annotation process is executed as follows. First, each sentence in the text is split to some bunsetsu-phrase chunks ${ }^{1}$, as shown in Figure 1 ("/" indicates a bunsetsu-phrase chunk boundary). Second, for each bunsetsu-phrase, an annotator finds the segment which represents a head element of an event,

\footnotetext{
${ }^{1}$ The bunsetsu-phrase is one of the fundamental units in Japanese, which consists of a content word (noun, verb, adjective, etc.) accompanied by some function words (particles, auxiliaries, etc.).
} 
and he/she adds the head tag to the segment (see also $h_{e a d}$ and head $_{2}$ in Figure 1). If the event has any other elements in addition to head element, the annotator also adds the mod tags to the segments representing modifiers to the head element $\bmod _{1}$ and $\bmod _{2}$ in Figure 1). The elements marked with any tags which have a common suffix number are constituents of the same event: that is, the elements marked with head 1 and $\bmod _{1}$ tags are constituents of $e_{1}$ and the elements marked with $h^{2} a d_{2}$ and $\bmod _{2}$ are constituents of $e_{2}$. Finally, the annotator adds the causal_rel tag between head 1 and head 2 as link information which indicates that the corresponding two events are held in a causal relation.

When there are any cue phrase markers helpful in recognizing causal relations such as ため (because) in (1a), the annotator also adds the marker tag to their segments.

\subsection{Annotation criteria}

To judge whether two events represented in text are held in a causal relation or not, we apply new criteria based on linguistic test.

The linguistic test is a method for judging whether target linguistic expressions conforms to a given set of rules. In our cases, the target expressions are two sets of bunsetsu-phrase chunks. Each set represents as a whole an event which can be an argument in a causal relation, such as in (2b) and (2c). The rules are realized as linguistic templates which are linguistic expressions including several slots.

In practice, a linguistic test is usually applied using the following steps:

1. Preparing a template.

2. Embedding the target expressions in the slots of the template to form a candidate sentence.

3 . If the candidate sentence is syntactically and semantically correct, the target expressions are judged to conform to the rules. If the candidate sentence is incorrect, the targets are judged non-conforming.

In this work, we prepared eighteen linguistic templates such as in Figure 2. The square brackets indicate the slots. The symbol $\langle\mathrm{adv}\rangle$ is replaced by one of three adverbs しばしば (often), 大抵 (usually), or 常に (always).

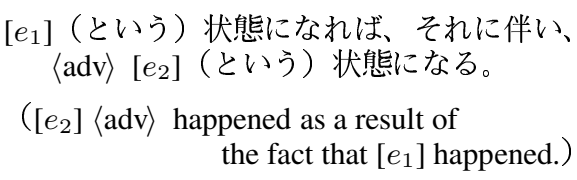

Figure 2: An example of linguistic templates

We embed two target expressions representing events in the slots of the template to form a candidate sentence. Then, if an annotator can recognize that the candidate sentence is syntactically and semantically correct, the causal relation is supposed to hold between two events. In contrast, if recognized that the candidate sentence is incorrect, this template is rejected, and the other template is tried. If all eighteen templates are rejected by the annotator, it is supposed that there is no causal relations between these two events. Note that the annotator's recognition of whether the candidate sentence is correct or incorrect, in other words, whether a causal relation is held between the two events embedded in the candidate sentence or not, is not really relevant to the author's intention.

The fundamental idea of our criteria based on linguistic test is similar to that of the criteria for annotation of implicit connectives adopted in PDTB corpus $^{2}$. In the annotation process of the PDTB corpus, an annotator judges whether or not the explicit connective, for example, "because", relates two linguistic expressions representing events. This process is essentially the same as ours.

Three adverbs in the linguistic templates, しばし ば (often), 大抵 (usually) and 常に (always), indicate a pragmatic constraint on the necessity of the relationship between any two events; the relations indicated by these words usually have a high degree of necessity. With this pragmatic constraint, we introduce an attribute to the causal_rel tags about the degree of necessity. For each of eighteen templates, if one judges the two target expressions as holding a causal relation by using the template with one of three adverbs, the necessity attribute value is added to the relation instance. If one judges the two target expressions as holding a causal relation by using the template deleting $\langle\mathrm{adv}\rangle$, three adverbs, the chance

\footnotetext{
${ }^{2}$ For detail instructions of the annotation criteria in PDTB corpus, see http://www.cis.upenn.edu/ pdtb/ manual/pdtb-tutorial.pdf.
} 
attribute value is added.

We assume that a target expression embedded in the slot is represented by a single sentence. If an event is represented by noun phrase (NP), the following rewriting rules are applied before embedded to the slot to transform the NP into a single sentence.

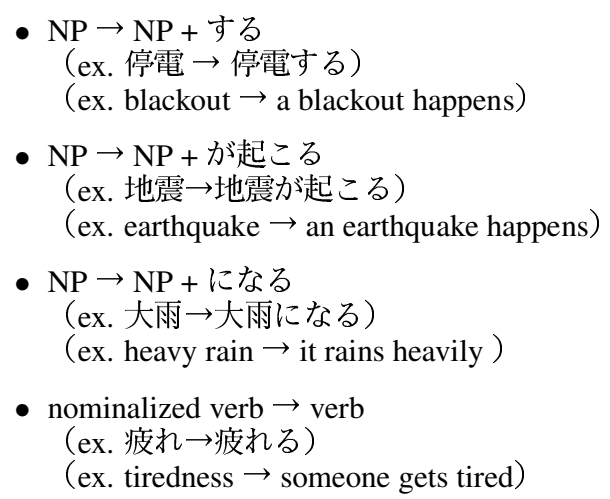

If a head element of a target expression representing an event is conjugated, the head element is replaced by its base form before embedded to the slot.

\subsection{Annotation ranges}

Ideally, we should try to judge for tagging of the causal relation tags over all any event pairs in text. However, it seems that the more the distance between two events represented in text, the smaller the probability of holding a causal relation between them. Thus, we set a constraint on the ranges of judgements. If both two events are represented in the same sentence or two sentences adjacent to each other, we try judgements, if not, skip judgements. This constraint is applied only when tagging the head tag. A modifier and its head element are sometimes located in different sentences overtly in Japanese text when anaphora or ellipsis phenomenon occurs. In such cases, we add mod tags to the text segments anywhere in the text.

\section{Data}

We selected as text for annotation Mainichi Shimbun newspaper articles (Mainichi, 1995). In particular, we used only articles included on the social aspect domain. When adding the causal relation tags to the text, it is preferable that each annotator can understand the whole contents of the articles. The contents of social aspect domain articles seems to be familiar to everybody and are easier to understand than the contents of articles included on politics, economy domain, etc.

Furthermore, in our previous examination, it is found that as the length of articles gets longer, it is getting hard to judge which bunsetsu-phrase chunks represent as a whole an event. This is because as described in Section 3.3, annotators sometimes need to search several sentences for modifiers of the head element in order to add mod tags precisely. Therefore, we focus on social aspect domain articles which consists of less than or equal to 10 sentences. After all, we extracted 750 articles (3912 sentences) for our annotation work with above conditions.

\section{Annotation workflow}

Three annotators have been employed. Each annotator has added tags to the same 750 document articles independently. Two annotators of the three are linguists, and the last one is the author of this paper. We denote each annotator under anonymity, $A, B$ and $C$. After training phase for annotators, we spent approximately one month to create a corpus annotated with causal relation information. The annotation workflow is executed efficiently using an annotation interface. Using the interface, all of annotators can add tags through only simple keyboard and mouse operations. The annotation workflow is as follows.

I. Annotation phase: A document article is displayed to each annotator. The sentences in the document are automatically split to bunsetsu-phrases by preprocessing. Some kinds of words such as connectives and verbs are highlighted to draw annotators' attention to the text segments which could represent elements in causal relation instances. The annotator finds text segments which represent causal relation instances, and then he/she adds the causal relation tags to their segments as described in Section 3.

II. Modification phase: After each annotator finished the annotation phase for a fixed number of document articles (in this work, 30 document articles), he/she moves to a modification phase. In this phase, first, only the segments with causal relation tags are extracted from the documents such as instances in Table 1. Then, 
Table 1: Examples of tagged instances

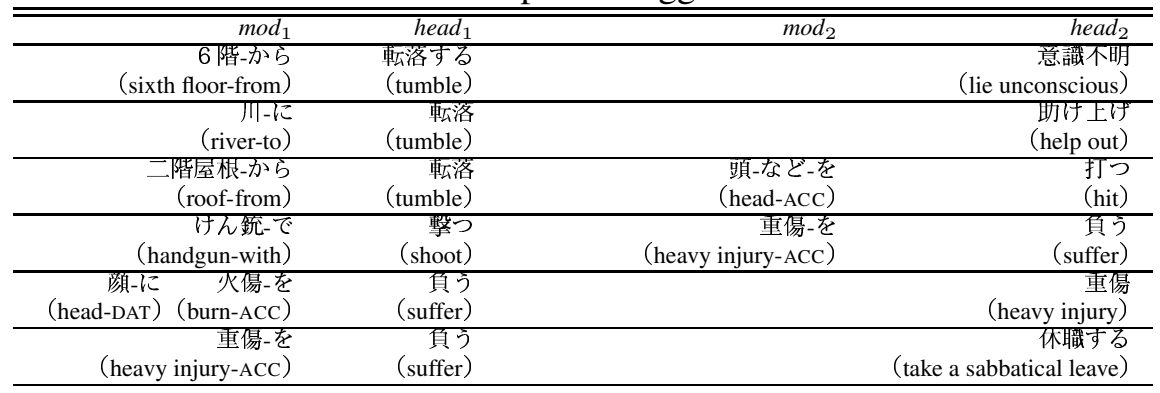

the same annotator who adds tags to the extracted segments, checks their extracted causal relation instances with attention. Since the extraction is done automatically, each annotator can check all the segments to be checked. When wrong tagged instances are found, they are corrected on the moment. After checking and correcting for all the extracted instances, the annotator moves back to the annotation phase in order to annotate a new 30 document articles set.

\section{Results}

\subsection{Total number of tagged instances}

2014 instances were tagged by the annotator $A, 1587$ instances by $B, 1048$ instances by $C$. Some examples of tagged instances are shown in Table 1.

The total numbers of tagged instances of the three annotators are quite different. Although all annotators tagged under the same annotation criteria, the annotator $A$ tagged to twice as many segments as the annotator $C$ did. Though this difference may be caused by some factors, we assume that the difference is mainly caused by missing judgements, since the annotators added tags to a variety of linguistic expressions, especially expressions without cue phrases.

To verify the above assumption, we again asked each annotator to judge whether or not a pair of linguistic expressions representing events is holding a causal relation. In this additional work, in order to prevent the annotators from skipping judgement itself, we present beforehand to the annotators the pairs of linguistic expressions to be judged. We presented a set of 600 pairs of linguistic expressions to each of the three annotators. All of these pairs are
Table 2: Inter-annotator agreement

\begin{tabular}{rrr|rrr}
\hline \hline$A$ & $B$ & $C$ & $\mathcal{S}_{\text {mixed }}$ & $\mathcal{S}_{n}$ & $\mathcal{S}_{c}$ \\
\hline 1 & 0 & 0 & 921 & 632 & 535 \\
0 & 1 & 0 & 487 & 487 & 255 \\
0 & 0 & 1 & 187 & 134 & 207 \\
\hline 1 & 1 & 0 & 372 & 230 & 90 \\
1 & 0 & 1 & 133 & 92 & 77 \\
0 & 1 & 1 & 140 & 107 & 83 \\
\hline 1 & 1 & 1 & 588 & 270 & 64 \\
\hline
\end{tabular}

the causal relation instances already tagged by one or more annotators in the main work described in the previous sections.

From the comparison between the results of the additional work and those of the main work, we found that if causal relation instances are expressed without explicit cues in text, they tend to be more frequently missed than those with explicit cues. The missing judgements on expressions without explicit cues are an important issue in the realization of more sophisticated analyses.

\subsection{Inter-annotator agreement}

We examined inter-annotator agreement. First, we define an agreement measure between two relation instances. Let $x$ and $y$ be causal relation instances tagged by two different annotators. The in stance $x$ consists of $e_{1 x}$ and $e_{2 x}$, and $y$ consists of $e_{1 y}$ and $e_{2 y}$. The event $e_{1 x}$ has $h_{e a d}$ as its head element. Similarly, head $2 x$, head $1 y$ and head $2 y$ are the head elements corresponding respectively to events $e_{2 x}, e_{1 y}$ and $e_{2 y}$. Then, we regard two instances $x$ and $y$ as the same instance, when head $1 x$ and head $1 y$ are located in the same bunsetsu-phrase and head $2 x$ and head $_{2 y}$ are also located in the same bunsetsuphrase. Using the above defined agreement measure, 
we counted the number of instances tagged by the different annotators.

Table 2 shows the results. The symbol " 1 " in the left-hand side of Table 2 indicates that the corresponding annotator tagged to instances, and the " 0 " indicates not tagged. For example, the fourth row ("110") indicates that both $A$ and $B$ tagged to instances but $C$ did not.

Let $\mathcal{S}_{\text {mixed }}$ denote a set of all tagged instances, $\mathcal{S}_{n}$ denote a set of all tagged instances with the necessity attribute value, and $\mathcal{S}_{c}$ denote a set of all tagged instances with the chance attribute value.

First, we focus on the relation instances in the set $\mathcal{S}_{\text {mixed }}$. The $1233(=372+133+140+588)$ instances are tagged by more than one annotator, and the 588 instances are tagged by all three annotators. Next, we focus on the two different contrastive sets of instances, $\mathcal{S}_{n}$ and $\mathcal{S}_{c}$. The ratio of the instances tagged by more than one annotator is small in $\mathcal{S}_{c}$. This becomes clear when we look at the bottom row ("111"). While the 270 instances are tagged by all three annotators in $\mathcal{S}_{n}$, only the 64 instances are tagged by all three annotators in $\mathcal{S}_{c}$.

To statistically confirm this difference, we applied the hypothesis test of the differences in population rates. The null hypothesis is that the difference of population rate is $d \%$. As a result, the null hypothesis was rejected at 0.01 significance level when $d$ was equal or less than 7 ( $p$-value was equal or less than 0.00805). In general, it can be assumed that if a causal relation instance is recognized by many annotators, the instance is much reliable. Based on this assumption and the results in Table 2, reliable instances are more concentrated on the set of instances with the necessity attribute value than those with the chance attribute value.

\section{Discussion}

In this section, we discuss some characteristics of in-text causal relations and suggest some points for developing the knowledge acquisition methods for causal relations. Here, to guarantee the reliability of the data used for the discussion, we focus on the $699(=230+92+107+270)$ instances marked by more than one annotator with the necessity attribute value. We examined the following three parts: (i) cue phrase markers, (ii) the parts-of-speech of head elements, and (iii) the positions of head elements.
Table 3: The number of instances with/without cue phrase markers

\begin{tabular}{ll}
\hline \hline with marker & 219 \\
without marker & 480 \\
\hline
\end{tabular}

Table 4: Cue phrase markers marked by annotators

\begin{tabular}{llr}
\hline \hline marker & & frequency \\
\hline ため & (because) & 120 \\
で & (by) & 35 \\
結果 & (result of) & 5 \\
ので & (because) & 5 \\
と & (when) & 5 \\
場合 & (when) & 4 \\
ば & (if) & 4 \\
ことから & (from) & 4 \\
から & (from) & 3 \\
\hline
\end{tabular}

\subsection{Cue phrase markers}

While annotating the document articles with our causal relation tags, head, mod, and causal_rel, the annotators also marked the cue phrase markers for causal relations with the marker tag at the same time. We investigated a proportion of instances attached with the marker tag.

The result is shown in Table 3. Table 4 shows the cue phrase markers actually marked by at least one annotator ${ }^{3}$.

It has been supposed that causal relation instances are sometimes represented with no explicit cue phrase marker. We empirically confirmed the supposition. In our case, only $30 \%$ of our 699 instances have one of cue phrase markers shown in Table 4, though this value can be dependent of the data.

This result suggests that in order to develop knowledge acquisition methods for causal relations with high coverage, we must deal with linguistic expressions with no explicit cue phrase markers as well as those with cue phrase markers.

\subsection{The parts-of-speech of head elements}

Next, we classified the events included in the 699 instances into two syntactic categories: the verb phrase (VP) and the noun phrase (NP). To do this, we used morphological information of their head elements. If the part-of-speech of a head is verb or adjective, the event is classified as a verb phrase. If

\footnotetext{
${ }^{3}$ The cue phrase markers whose frequencies are less than three are not listed due to space limitation in Table 4.
} 
Table 5: The syntactic types

\begin{tabular}{llrr}
\hline \hline & & $e_{1}$ & $e_{2}$ \\
\hline VP & $\begin{array}{l}\text { (verb) } \\
\text { (adjective) }\end{array}$ & 365 & 412 \\
\hline NP & $\begin{array}{l}\text { (verbal noun) } \\
(\text { general noun) }\end{array}$ & 322 & 269 \\
\hline & others & 12 & 18 \\
\hline
\end{tabular}

the part-of-speech of a head is noun (including general noun and verbal noun), the event is classified as a noun phrase. We used ChaSen ${ }^{4}$ to get part-ofspeech information.

The result is shown in Table 5. More than half events are classified as the VP. This matches our intuition. However, the number of events classified as the NP is comparable to the number of events classified as the VP; 322 events of $e_{1}$ are represented as noun phrases, and 269 events of $e_{2}$ are also represented as noun phrases.

This result is quite suggestive. To promote the current methods for knowledge acquisition to further stage, we should develop a knowledge acquisition framework applicable both to the verb phrases and to the noun phrases.

\subsection{The positions of head elements}

For each $e_{1}$ and $e_{2}$ included in the 699 instances, we examined the positions of their head elements in the sentences.

We consider dependency structures between bunsetsu-phrases in the original sentences from which causal relation instances are extracted. The dependency structures form tree structures. The bunsetsuphrase located in the end of the sentence is the root node of the tree. We focus on the depth of the head element from the root node. We used CaboCha ${ }^{5}$ to get dependency structure information between bunsetsu-phrases.

The results are shown in Figure 3 and Figure 4. Figure 3 is the result for the head elements of $e_{1}$, and Figure 4 is the result for the head elements of $e_{2}$. The letter " $\mathrm{f}$ " in Figure 3 and Figure 4 indicates frequency at each position. Similarly, the letter "c"

\footnotetext{
${ }^{4}$ Available from http://chasen.aist-nara.ac. jp/hiki/ChaSen/.

${ }^{5}$ Available from http://chasen.org/ taku/ software/cabocha/.
}

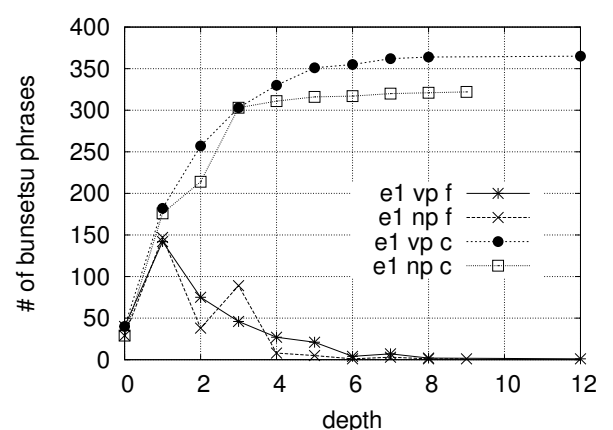

Figure 3: The positions of head elements $\left(e_{1}\right)$

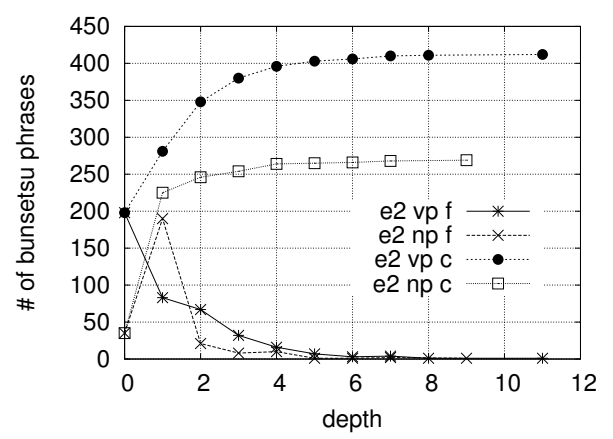

Figure 4: The positions of head elements $\left(e_{2}\right)$

indicates cumulative frequency.

In Figure 4, the 198 head elements of the events represented as a verb phrase are located in the end of the sentences, namely depth $=0$. The 190 of the 269 events represented as a noun phrase are located in depth $=1$. For events represented as either a verb phrase or a noun phrase, over $80 \%$ of head elements of the events are located within depth $<3$. In Figure 3, similarly, over $80 \%$ of head elements of the events are located within depth $<4$.

These findings suggest that the most of the events are able to be found simply by searching the bunsetsu-phrases located in the shallow position at the phase of causal knowledge acquisition.

\subsection{Relative positions of two head elements}

Finally, we examined relative positions between head elements of $e_{1}$ and $e_{2}$ where these two events are held in a causal relation. In Section 7.3, we discussed each absolute position for $e_{1}$ and $e_{2}$ by means of the notion of depth in sentences. Here, we focus on the difference $(D)$ of the depth values between $e_{1}$ and $e_{2}$.

The result is shown in Table 6 . The symbol " $e_{1} \Rightarrow$ $e_{2}$ " in Table 6 indicates the case where the head element of $e_{1}$ is located nearer to the beginning of the 
Table 6: Relative positions of two head elements

\begin{tabular}{l|r|rr}
\hline \hline & & $e_{1} \Rightarrow e_{2}$ & $e_{2} \Rightarrow e_{1}$ \\
\hline \multirow{4}{*}{ intra-sentential } & $D=1$ & 259 & 15 \\
& $=2$ & 152 & 23 \\
& $>2$ & 33 & 4 \\
\cline { 2 - 4 } & no dep & \multicolumn{2}{|c}{72} \\
\hline inter-sentential & & \multicolumn{2}{|c}{141} \\
\hline
\end{tabular}

sentence than that of $e_{2}$. The " $e_{2} \Rightarrow e_{1}$ " indicates the opposite case. The symbol "no dep" indicates the case where neither the condition $a$ nor $b$ is satisfied:

$a$. the head element of $e_{2}$ is an ancestor of the head element of $e_{1}$.

$b$. the head element of $e_{2}$ is a descendant of the head element of $e_{1}$.

The symbol "inter-sentential" indicates the case where two head elements appear in different sentences.

The most instances (259 instances) are categorized into $D=1$ on $e_{1} \Rightarrow e_{2}$, that is, the head element of $e_{1}$ directly depends on the head element of $e_{2}$. This result matches our intuition. However, there are several other cases. For example, 152 instances are categorized into $D=2$ on $e_{1} \Rightarrow e_{2}, 72$ instances are categorized into "no dep". Most of the instances extracted from sentences including any parallel relations are categorized into "no dep". In this study, we consider causal relation instances as binary relation. To deal with instances categorized into "no dep" adequately, we should extend our framework to the more complex structure.

\section{Conclusion}

We reported our causal relation tags and the annotation workflow. Using the annotated corpus, we examined the causal relation instances in Japanese text. From our investigation, it became clear that what amount of causal relation instances are present, where these relation instances are present, and which types of linguistic expressions are used for expressing these relation instances in text.

\section{Acknowledgement}

This research is supported by the 21COE Program "Framework for Systematization and Application of
Large-Scale Knowledge Resources" and the Grantin-Aid for Creative Basic Research (13NP0301) "Language Understanding and Action Control". We would like to express our special thanks to Junji Etoh, Yoshiko Ueda, Noriko Sogoh, and Tetsuro Takahashi for helping us to create our corpus. We are grateful to the reviewers for their suggestive comments.

\section{References}

B. Altenberg. 1984. Causal linking in spoken and written English. Studia Linguistica, 38:1.

R. Girju. 2003. Automatic detection of causal relations for question answering. In Proc. of the 41st ACL, Workshop on Multilingual Summarization and Question Answering.

T. Inui. 2004. Acquiring causal knowledge from text using connective markers. Ph.D. thesis, Graduate School of Information Science, Nara Institute of Science and Technology.

Y. Liu. 2004. Semantics and usages of connectives for causal relations in modern Japanese - cases of 'dakara', 'sitagatte', 'soreyue(ni)', 'sonokekka', 'sonotame(ni)' -. Ph.D. thesis, The Graduate School of Languages and Cultures, Nagoya University.

Mainichi. 1995. Mainichi Shimbun CD-ROM version.

D. Marcu. 1997. The rhetorical parsing, summarization, and generation of natural language texts. Ph.D. thesis, Department of Computer Science, University of Toronto.

E. Miltsakaki, R. Prasad, A. Joshi, and B. Webber. 2004. Annotating discourse connectives and their arguments. In Proc. of the HLT/NAACL Workshop on Frontiers in Corpus Annotation.

S. Nishizawa and Y. Nakagawa. 1997. A method of discourse structure understanding in Japanese task-free conversation for causal conjuction. Natural Language Processing, 4(4):61-72. (in Japanese).

M. Palmer, D. Gildea, and P. Kingsbury. 2005. The proposition bank: A corpus annotated with semantic roles. Computational Linguistics Journal, 31(1).

J. Pustejovsky, J. M. Castaño, R. Ingria, R. Sauri, R. J. Gaizauskas, A.Setzer, G. Katz, and D. R. Radev. 2003. TimeML: Robust specification of event and temporal expressions in text. In New Directions in Question Answering, pages 28-34.

H. Sato, K. Kasahara, and K. Matsuzawa. 1999. Rertrieval [sic] of simplified causal knowledge in text and its application. In Technical report of IEICE, Thought and Language. (in Japanese). 